\title{
Effect of Ageing on the Martensitic Transformation in a Monocrystalline Cu-Al-Ni Shape Memory Alloy
}

\author{
V. Recarte, M.L. Nó*, J. San Juan \\ Dpto. Física Mat. Condensada, Fac. Ciencias, Univ. Pais Vasco, Apdo 644, 48080 Bilbao, Spain \\ * Dpto. Física Aplicada II, Fac. Ciencias, Univ. Pais Vasco, Apdo. 644, 48080 Bilbao, Spain
}

\begin{abstract}
In this work we have studied the effect of post quench ageing on the martensitic transformation in a monocrystalline $\mathrm{Cu}-13,7 \mathrm{Al}-5 \mathrm{Ni}$ (wt.\%) shape memory alloy. Internal friction and modulus change have been measured by an inverted torsion pendulum operating at $1 \mathrm{~Hz}$ for different ageing times. The results show a shift of the heating and cooling internal friction peaks towards high temperatures with the increase of the ageing time at $473 \mathrm{~K}$. On the other hand, the quenched sample shows only one sharp peak on heating and cooling runs with low hysteresis transformation. Nevertheless, two separated internal friction peaks appears on heating as ageing time increases. The results can be attributed to a change in the thermally formed martensite from $\beta_{1}{ }^{\prime}$ in the quenched sample to a mixture of $\beta_{1}{ }^{\prime}$ and $\gamma_{1}{ }^{\prime}$ in the aged sample.
\end{abstract}

\section{INTRODUCTION}

In $\mathrm{Cu}-\mathrm{Al}-\mathrm{Ni}$ shape memory alloys the crystal structure of thermally induced martensite phases varies with the alloy composition (1). There is a composition range in wich two martensites, $\beta_{1}{ }^{\prime}$ and $\gamma_{1}{ }^{\prime}$, coexist for different thermal treatments $(2,3)$. On the other hand it has been found that crystal structures of martensite phases change with heat treatment for a given composition, either varying the quenching rate (4) or ageing at different temperatures (5). But there is very few studies about the evolution with ageing of the transformation sequence of the $\beta_{1}{ }^{\prime}$ and $\gamma_{1}{ }^{\prime}$ marensites. In fact, the only work in our knowledgement that studies the kinetic of the transition between $\beta_{1}{ }^{\prime}$ and $\gamma_{1}$ 'martensites has been done for short ageing time (about 2 hours) (5). Nevertheless the study of this evolution between $\beta_{1}{ }^{\prime}$ and $\gamma_{1}{ }^{\prime}$ martensites for very long ageing time is an important aspect, from a technological point of vew, in order to obtain a good and reliable behaviour of these alloys until $473 \mathrm{~K}(6)$.

In this work we have studied the effect of post quench ageing at $473 \mathrm{~K}$ on a monocrystalline $\mathrm{Cu}-13,7$ Al-5 $\mathrm{Ni}\left(\mathrm{wt} \%\right.$ ) shape memory alloy that undergoes a thermally induced double $\beta_{1}$ ' and $\gamma_{1}{ }^{\prime}$, martensitic transformation. We have used the internal friction techniques to carry out this work, because these techniques have shown to be very useful to study the martensitic transformation in shape memory alloys $(7,8,9)$. Besides the internal friction integral allows us to follow the transformed volume fraction evolution during the $\beta_{1}{ }^{\prime}-\gamma_{1}$ transition that has not been studied up today.

\section{EXPERIMENTAL METHODS}

Monocrystalline samples of a $\mathrm{Cu}-\mathrm{Al}-\mathrm{Ni}$ alloy with a nominal composition $\mathrm{Cu}-13,7 \mathrm{Al}-5 \mathrm{Ni}$ mass \% have been used. To carry out the measurements, $0.85 * 5 * 50 \mathrm{~mm}$ samples were cut using a low speed diamond saw. In order to retain the $\beta$ phase, they were annealed at $1173 \mathrm{~K}$ during 30 minutes and quenched into water at $363 \mathrm{~K}$. 
Simultaneous internal friction, modulus and microdeformation measurements have been carried out in an inverted torsion pendulum operating at $1 \mathrm{~Hz}$ in a temperature range between $80 \mathrm{~K}$ and $750 \mathrm{~K}(10)$ ). A heating rate of $60 \mathrm{~K}^{*} \mathrm{~h}^{-1}$ and an oscillation amplitude of $\varepsilon_{\mathrm{m}}=2 * 10^{-5}$ have been used for the measurements. The samples have not been dismounted when studying the transformation evolution with post quench heat treatment. In situ ageing has been carried out in the pendulum.
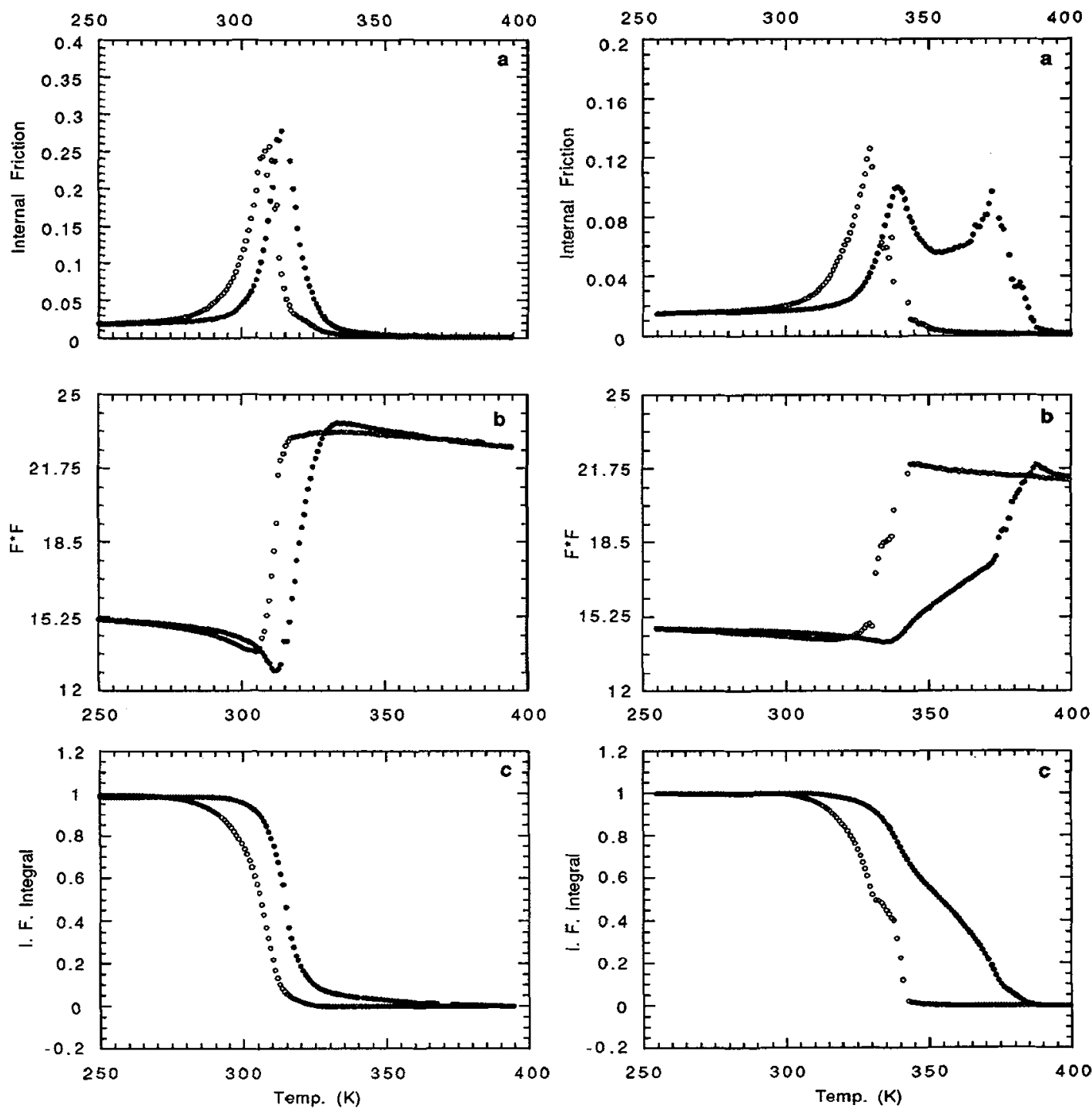

Fig. 1. Measurements during heating and cooling run for the sample in the quenched state. (a) and (b) show the internal friction spectra and the corresponding modulus measurements. The integral curve of the internal friction spectra is shown in (c).

Fig. 2. Measurements during heating and cooling run for the sample aged 20 hours at $473 \mathrm{~K}$. (a) and (b) show the internal friction spectra and the corresponding modulus measurements. The integral curve of the internal friction spectra is shown in (c). 


\section{EXPERIMENTAL RESULTS}

Fig. 1-a and fig. 1-b show the internal friction spectra and the corresponding modulus measurements for the quenched state. The integral curve of internal friction is shown in fig. 1-c. The integral curves are normalized separately for each run in temperature, either cooling or heating. The internal friction curve shows only a peak for both the direct and reverse transformation. Equally, there is only a fall in modulus during the martensitic transformation and the integral curve shows only a stage.

The same curves for the sample aged 20 hours at $473 \mathrm{~K}$ can be seen in fig. 2 -a-b-c respectively. The internal friction spectrum, fig 2 -a, shows only a peak during the direct transformation but two separated peaks during the reverse transformation. On the other hand, the modulus measurement fig 2-b shows two consecutive falls during direct transformation. In the reverse transfomation these two falls appear separated in temperature. We can see two different stages during the martensitic transformation $\left(\mathrm{P}_{1}\right.$ for the low temperature peak and $\mathrm{P}_{2}$ for the high temperature peak) that overlap during the direct transformation and that are separated during the reverse transformation. The integral curve of the internal friction spectrum for the sample aged at $473 \mathrm{~K}$, fig $2-\mathrm{c}$, shows clearly this difference in hysteresis for the two peaks.
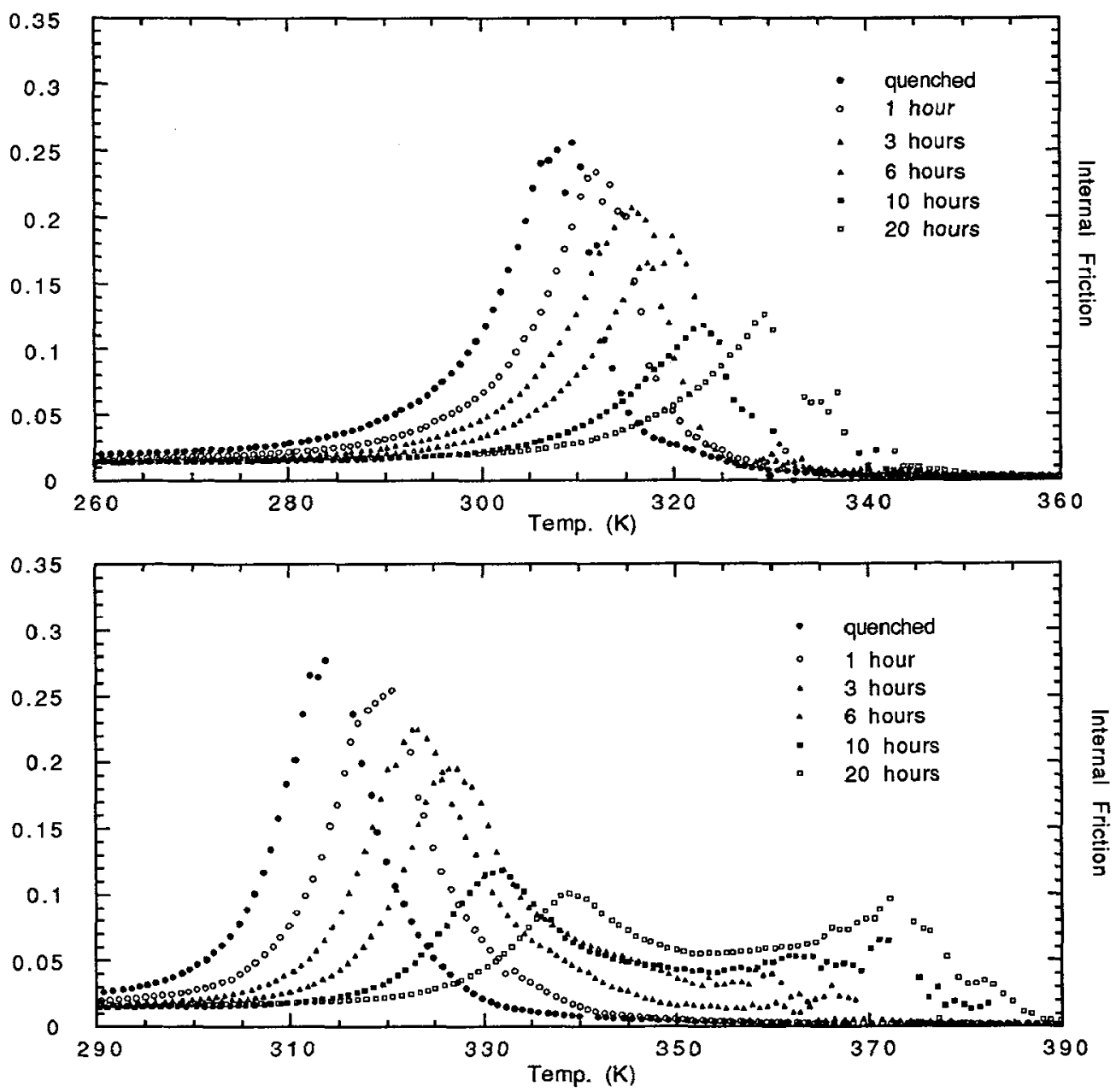

Fig. 3. Evolution of the internal friction spectra with the ageing time for the direct transformation (a), and the reverse transformation (b). 
Fig. 3 shows the evolution of internal friction spectra with the ageing time for the direct transformation, fig. 3-a, and the reverse transformation, fig. 3-b. There is a general increase in the peak temperatures with the ageing time. On the other hand, the height of the high temperature $\mathrm{P}_{2}$ peak increases with treatment, in contrast with the decrease of the height of $P_{1}$ peak. There is a high level of internal friction between the two peaks during the reverse transformation wich must indicate that transformation does not stop. The $\mathrm{P}_{1}$ peak shows a low experimental dispersion, opposite to the behaviour of the $\mathrm{P}_{2}$ peak that shows a high dispersion in measurement. This fact indicates a smooth and continous character for the low temperature transformation and a jerky behaviour of the high temperature transformation.

Fig. 4-a shows the evolution with ageing time of characteristic temperatures $A_{S 1}$ and $M_{f 1}$ of the low temperature transformation. The temperatures $A_{f 2}$ and $M_{S 2}$ associated to the high temperature transformation are shown in fig. 4-b. These temperatures have been obtained from the integral curves of the internal friction spectra. In all the cases there is a change in transformation temperatures that after an hour of ageing begin to increase with the treatment time. The evolution of the two transformation hysteresis is obtained from the expressions $\mathrm{H}_{1}=\mathrm{A}_{\mathrm{S} 1}-\mathrm{M}_{\mathrm{f} 1}$ and $\mathrm{H}_{2}=\mathrm{A}_{\mathrm{f} 2}-\mathrm{M}_{\mathrm{S} 2}$. The hysteresis increases at the same rate for both transformations with the ageing time, fig. 5 .

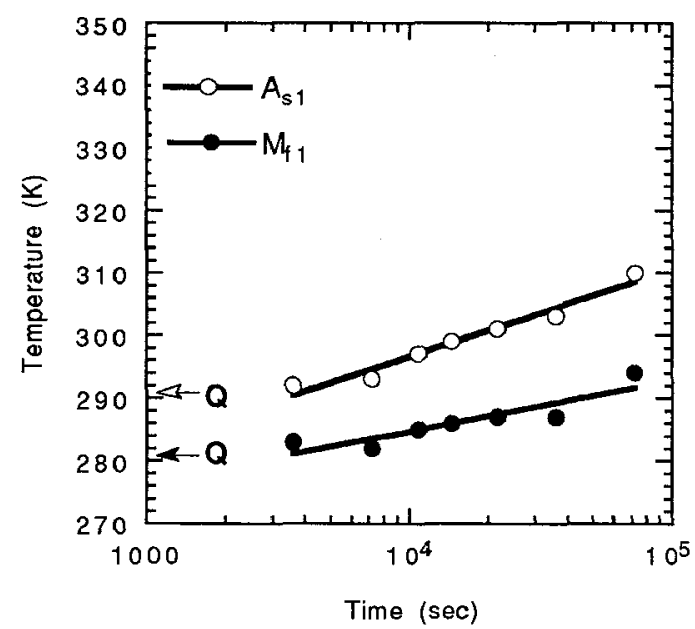

Fig 4-a. Evolution of characteristic temperatures $A_{\mathrm{S} 1}$ and $M_{\mathrm{f} 1}$ with ageing time. The temperatures for the quenched state are indicated by arrows.

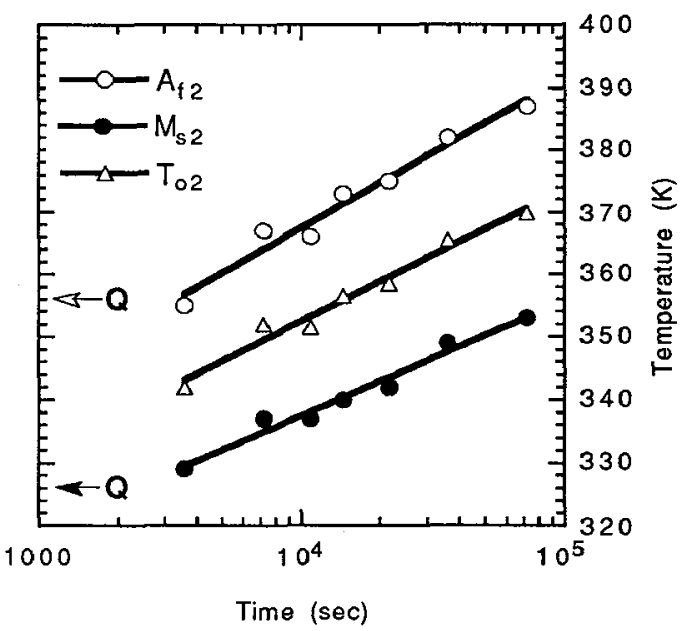

Fig 4-b. Evolution of characteristic temperatures $A_{f 2}$ and $M_{S 2}$ with ageing time. The temperatures for the quenched state are indicated by arrows.

\section{DISCUSSION}

This alloy shows a double transformation of two martensitic phases $\beta_{1}^{\prime}$ and $\gamma_{1}$ thermally induced. The low temperature $P_{1}$ peak shows the characteristics of a $\beta-\beta_{1}^{\prime}$ transformation: low hysteresis $\left(\approx 10^{\circ} \mathrm{C}\right)$ and a smooth behaviour. On the contrary, the high temperature $\mathrm{P}_{2}$ peak shows a higer hysteresis $\left(\approx 30^{\circ} \mathrm{C}\right)$ and a jerky behaviour characteristic of a $\beta-\gamma_{1}^{\prime}$ transformation.

During cooling both transformations are produced successivelly but almost simultaneously, arising only one internal friction peak independently of the ageing time at $473 \mathrm{~K}$ ( fig. 1-a and fig. 2-a ). During heating, the different hysteresis of both kind of transformations $\beta-\beta_{1}{ }_{1}$ and $\beta-\gamma_{1}^{\prime}$ produces a splitting of the internal friction peaks during the reverse transformations $\beta_{1}^{\prime}-\beta$ and $\gamma_{1}{ }_{1}-\beta$ ( fig. 2 -a ). Nevertheless we have to point out that the modulus curve in fig. $2-b$ and the integral curve in fig. 2-c measured during cooling show two clearly different stages that we can attribute to $\beta-\gamma_{1}^{\prime}$ and $\beta-\beta_{1}^{\prime}$ transformations.

In fact, this splitting of the internal friction peaks and the double behaviour of the transformation is developped more and more with the increase of the ageing time, fig. 3 . The evolution of the internal friction peaks plotted in fig. 3-b allows us to conclude that the ageing treatment at $473 \mathrm{~K}$ produces an increase of the volume fraction of the transformed $\gamma_{1}$ phase. Indeed, we observe an evolution from a transformation 
mainly of a $\beta-\beta_{1}^{\prime}$ kind in the quenched sample to a mixed transformation of $\beta-\gamma_{1}$ and $\beta-\beta_{1}^{\prime}$ at the end of the 20 hours at $473 \mathrm{~K}$ treatment ( fig. 3-b ).

The increase of the transformation temperatures with ageing time could be attributed in a first approach to two kind of processes:

a) The precipitation of the stable $\gamma_{2}$ phase.

b) An ordering process of the metastables phases.

A precipitation process of the $\gamma_{2}$ phase should produce a poorer matrix in $\mathrm{Al}$ and consequently an increase of the transformation temperatures. Nevertheless, in the $\mathrm{Cu}-\mathrm{Al}$ alloys $(11)$ and in the $\mathrm{Cu}-\mathrm{Al}-\mathrm{Ni}$ alloys (12) the thermally induced martensites are produced in a $\alpha^{\prime}, \beta^{\prime}, \gamma^{\prime}$ sequential order with the increase of the aluminium concentracion. Consequently, a matrix more and more poor in aluminium should promote the formation of $\beta^{\prime}$ martensite in contradiction with the experimental results.

Besides, the internal friction peaks area is associated to the transformed volume fraction (13) and in our case, the integral of the internal friction spectra obtained during the reverse transformation in the quenched sample and after 20 hours ageing at $473 \mathrm{~K}$ shows almost the same value within an error of $5 \%$. This result means that we have not a decrease of the total transformed fraction and is not in agreement with the presence of the precipitated $\gamma_{2}$ phase, required to shift the transformation temperatures.

On the other hand, an ordering process should increase the transformation temperatures if some stabilization of the martensites is produced. In this case, in the $\mathrm{Cu}-\mathrm{Al}-\mathrm{Ni}$ alloys the $2 \mathrm{H}$ structure $\left(\gamma_{1}{ }^{\prime}\right)$ will be more easily stabilized than the $18 \mathrm{R}$ structure $\left(\beta_{1}^{\prime}\right)(4)$. So, the evolution of transformation temperatures could be attributed in our case to an ordering process.

With the ageing time a quicker increase of the $M_{f 2}$ and $A_{\mathrm{s} 2}$ than the $M_{\mathrm{s} 1}$ and $A_{f 1}$ temperatures produces a widening of the temperature interval between both transformation peaks such as can be observed from the internal friction spectra. This result is in agreement with an ordering process that favours an earlier nucleation of the $\gamma_{1}$ ' phase with ageing. This way a higher volume fraction of $\gamma_{1}$ ' is transformed before the start of the $\beta_{1}{ }_{1}$ nucleation and as a consequence the peak area associated to the $\gamma_{1}$ transformation increases with the ageing time, while the peak area associated to the $\beta^{\prime}{ }_{1}$ transformation decreases.

Also, the fact that the nucleation and the further transformation of the $\beta_{1}^{\prime}$, takes place in a matrix with a complex stress field due to the previously transformed $\gamma_{1}$ should modifie the elastic term of the transformation and consequently the hysteresis of the transformation. Indeed, the high level of the internal friction for the longer ageing times, when both peaks become very separated, indicates that even in this case the $\gamma_{1}-\beta$ transformation starts before that the $\beta_{1}^{\prime}-\beta$ transformation finishes. This behaviour strengthens the idea of a complex stress field having locally equilibrium conditions.

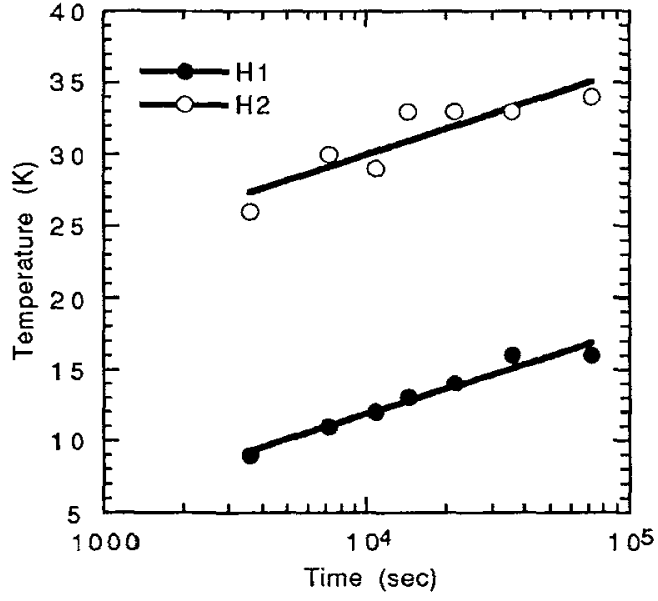

Fig. 5. Influence of ageing time on the hysteresis transformation $\mathrm{H}_{1}$ and $\mathrm{H}_{2}$.

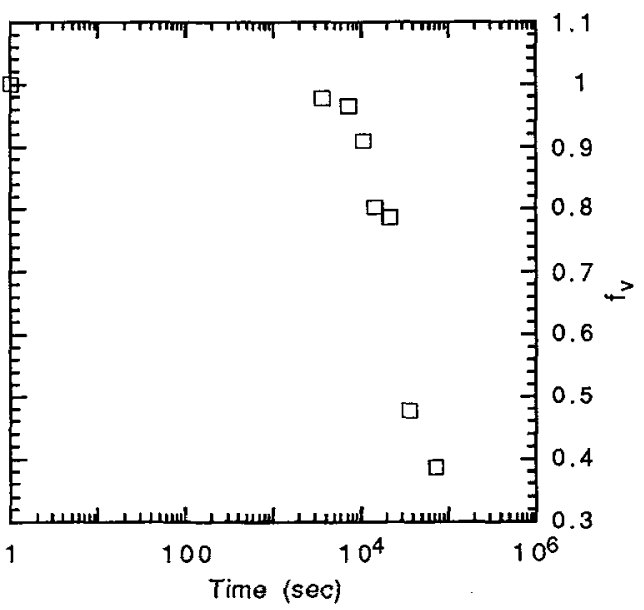

Fig. 6. Evolution of volume fraction $\left(f_{v}\right)$ of $\beta_{1}^{\prime}$ phase with ageing time, normalized from the quenched state. 
Obviously, the presence of a mixed $\gamma_{1}$ and $\beta_{1}^{\prime}$ transformation produces a loss of thermoelasticity due to a more difficult autoaccommodation of the different kinds of martensite and justify the increase of the hysteresis (fig.5) as well as some irreversibility of the transformation. This irreversibility has also been observed by several authors (14) and attributed to the retained $\beta$ phase (15) and to the plastic deformation produced during the mixed transformation (16).

Nevertheless, we have to remark that initially we start with a sample that after quenching undergoes a simple $\beta-\beta_{1}^{\prime}$ transformation showing only an internal friction $P_{1}$ peak. Along ageing a decrease of the volume fraction ( $f_{v}$ ) of the transformed $\beta_{1}^{\prime}$ phase is observed (fig 6 ) from the evolution of the $P_{1}$ peak area that is counterbalanced by an increase of the transformed $\gamma_{1}^{\prime}$ phase. This way, the behaviour of the transformation evolves with ageing from a simple $\beta-\beta_{1}^{\prime}$ transformation towards a mixed $\beta-\beta_{1}^{\prime}$ plus $\beta-\gamma_{1}^{\prime}$ transformation.

If we take the temperature $T_{02}=1 / 2 *\left(M_{\mathrm{S} 2}+A_{f 2}\right)(17)$ for the $\beta-\gamma_{1}$ transformation, that is developed with ageing, we observe that $T_{\mathrm{O} 2}$ increases with the ageing time (fig. 4-b). This means that along ageing the shifting of the whole hysteresis cycle should be linked to a process of chemical origin, like the proposed ordering.

\section{CONCLUSIONS}

We have shown that in the concentration range in which both kind of martensites $\beta_{1}{ }^{\prime}$ and $\gamma_{1}{ }^{\prime}$ can coexist, a long time evolution between both martensites takes place during ageing at $473 \mathrm{~K}$. The volume fraction of both martensites evolves along ageing for a time longuer than $10^{5}$ seconds, modifiying the behaviour of the alloy during the martensitic transformation.

\section{ACKNOWLEDGEMENT}

This work has been carrried out thanks to the financial support of the Spanish "Comision Interministerial de Ciencia y Tecnología CICyT " in the frame of the project MAT 92-0353

\section{REFERENCES}

(1) Vasilenko A. Y., Sal'nikov V. A., Kosilov A. T., Phys. Metals 4 (1982) 694

(2) Friend C. M., Ortín J., Planes A., Mañosa Ll., Yoshikawa M., Scripta Metall. 24 (1990) 1641

(3) Recarte V., San Juan J., Herreros J., Nó M. L., Journal de Physique IV 1 (1991) C4-271

(4) Sakamoto H., Shimizu K., ISIJ International 29 (1989) 395

(5) Van Humbeeck J., Van Hulle D., Delaey L., Ortín J., Seguí C., Torra V., Trans JIM 28 (1987) 383

(6) Delaey L., Materials Science and Technology Vol.5, pag 339-404 Ed. by R. W. Cahn, P. Haasen, E. J. Kramer, VCH Verlagsgesellchaft (Weinheim) 19991

(7) De Batist R., Proc. of $M^{3} D$, pag.45-59, Ed. by V. K. Kinra and A. Wolfenden, ASTM publications 1992

(8) Van Humbeeck J., Proc. of Summer School of Internal Friction in Solids, pag. 131-149,Ed. S. Gorczyca, L. B. Magalas, AGH publications (Cracow)

(9) Van Humbeeck J., Proc. of ICIFUAS-9, pag. 337-344, Ed. by T. S. Kê, Pergamon Press (1990)

(10) Oleaga A., Hurtado I., Nó M. L., Esnouf C., San Juan J., Anales de Física B 85 (1989) 373

(11) Swann P. R., Warlimont H., Acta Met. 11 (1963) 1099

(12) Friend C. M., Scripta Metall. 23 (1989) 1817

(13) Pérez-Sáez R. B., Master Thesis Univ. País Vasco (1993)

(14) Seguí C. , Cesari E., Van Humbeeck J., Mater. Trans. JIM 5 (1990) 375

(15) Yang J. H., Zhang C. S., Zhao L. C., Lei T. C., Scripta Metall. 21 (1987) 259

(16) Yang J. H., Liu C., Zhao L., Lei T., Proc. Shape Memory Alloys 86, China Academic Publishers (1986) 327

(17) Funakubo H., "Shape Memory Alloys", Precision Machinery and Robotics Vol. 1. Gordon and Breach Science Publishers (1987) New York 